\title{
Costos por ausentismo laboral atribuibles al consumo de tabaco en el Instituto Mexicano del Seguro Social y en México, 2006-2009
}

\author{
Carlos Manuel Guerrero-López, Lic en Econ, (I) Luz Myriam Reynales-Shigematsu, D en C, (I) \\ Jorge Alberto Jiménez-Ruiz, M en Econ, ${ }^{(1)}$ Roberto Karam-Araujo, MC,(2) \\ César Augusto Maldonado-Cruz, Ing, ${ }^{(2)}$ Rafael Camacho-Solís, MC. ${ }^{(2)}$
}

\author{
Guerrero-López CM, Reynales-Shigematsu LM, \\ Jiménez-Ruiz JA, Karam-Araujo R, \\ Maldonado-Cruz CA, Camacho-Solis R. \\ Costos por ausentismo laboral atribuibles \\ al consumo de tabaco en el Instituto Mexicano \\ del Seguro Social y en México, 2006-2009. \\ Salud Publica Mex 20 2;54:233-24I.
}

\begin{abstract}
Resumen
Objetivo. Calcular los costos por ausentismo laboral atribuibles al tabaquismo en los asegurados del IMSS y población ocupada en México para el periodo 2006-2009. Material y métodos. Los costos se estimaron usando fracciones atribuibles para cáncer pulmonar, enfermedad cerebrovascular, enfermedad pulmonar obstructiva crónica e infarto agudo de miocardio, así como registros de salarios y días de incapacidad por esas enfermedades en asegurados del IMSS. Los parámetros encontrados se extrapolaron a la población ocupada. Resultados. El IMSS erogó I 43.9 millones de pesos a precios de 2009 atribuibles al tabaquismo como subsidio por incapacidad en el período 2006-2009. La productividad perdida atribuible al tabaquismo en asegurados del IMSS ascendió a 298.2 millones de pesos y a 437.8 millones de pesos en población ocupada del país en el mismo período. Conclusiones. El tabaquismo impone costos importantes a las instituciones de seguridad en el país y causa pérdidas en la productividad. Por tanto, deben fortalecerse las políticas gubernamentales de control del tabaco incluidas en el Convenio Marco para el Control del Tabaco.
\end{abstract}

Palabras clave: México; tabaquismo; ausentismo
Guerrero-López CM, Reynales-Shigematsu LM, Jiménez-Ruiz JA, Karam-Araujo R,

Maldonado-Cruz CA, Camacho-Solis R.

Absenteeism attributable to smoking

in the Mexican Social

Security Institute, 2006-2009.

Salud Publica Mex 2012;54:233-24I.

\begin{abstract}
Objective. To calculate the absenteeism costs by lung cancer, cerebrovascular disease, chronic obstructive pulmonary disease and acute myocardial infarction attributable to smoking in the Mexican Social Security Institute (IMSS) and the occupied population from 2006 to 2009. Materials and methods. Productivity loss data from selected illnesses were obtained from IMSS records. The smoking attributable fraction was used, and extrapolation to occupied population was conducted. Results. IMSS paid \$143.9 million pesos (2009 prices) attributable to smoking between 2006 and 2009. The productivity loss was $\$ 298.2$ million pesos and $\$ 437.8$ million pesos in the occupied population, attributable to smoking. Conclusions. Tobacco smoking implies costs to the individual, families and society, which urge to strengthen policies contained in the Framework Convention on Tobacco Control by the WHO.
\end{abstract}

Keywords: Mexico; smoking; absenteeism

(I) Departamento de Investigación sobre Tabaco, Centro de Investigación en Salud Poblacional, Instituto Nacional de Salud Pública. México.

(2) Instituto Mexicano del Seguro Social. México. 
$E_{p}^{1}$ tabaquismo es un problema prioritario de salud pública pues es una causa importante de enfermedad y discapacidad tanto en México como en el mundo. Las repercusiones del tabaquismo incluyen daños a la salud humana, costos directos (gastos en atención médica), costos indirectos (incapacidad, discapacidad y pérdida de productividad) y costos intangibles tales como sufrimiento, dolor y afectación a familiares, tanto en fumadores activos como en pasivos. ${ }^{1,2}$

Cuantificar las consecuencias económicas y financieras del tabaquismo resulta de gran utilidad para planear las políticas en salud pública en un contexto de escasez de recursos económicos, ya que el sistema de salud debe erogar una cantidad importante de recursos para atender las enfermedades relacionadas con el tabaquismo, que principalmente son cardiovasculares, respiratorias y neoplasias. ${ }^{3}$ En particular, las afectaciones en términos de pérdida de productividad no recaen solamente sobre el paciente, sino también tienen un efecto multiplicador en la planta productiva del país al afectar un valioso insumo, el capital humano.

Existen diversos enfoques y alcances para determinar los costos que una enfermedad impone. En nuestro estudio se aborda la cuantificación de costos institucionales, en este caso el IMSS, y los costos indirectos relativos al ausentismo laboral atribuibles al consumo de tabaco desde la perspectiva de capital humano, que básicamente consiste en considerar la pérdida de productividad como el valor de la producción perdida. ${ }^{4-7}$ Es decir, puede usarse el salario o remuneración del individuo como el costo de oportunidad de los días de ausencia laboral causados por una enfermedad. ${ }^{8}$

En este sentido, el presente estudio tiene los siguientes objetivos: 1) estimar los montos de subsidio erogados por el IMSS atribuibles al consumo de tabaco por incapacidades debidas a las cuatro principales enfermedades asociadas con el tabaquismo: cáncer pulmonar $(\mathrm{CP})$, enfermedad cerebrovascular $(\mathrm{ECV})$, enfermedad pulmonar obstructiva crónica (EPOC) e infarto agudo de miocardio (IAM); y 2) estimar los costos por ausentismo laboral causados por las enfermedades asociadas con el consumo de tabaco antes señaladas en asegurados del IMSS a través de una aproximación de capital humano. Asimismo, se realiza un ejercicio de extrapolación de los parámetros encontrados en la población del IMSS a fin de aproximar estos costos en la población ocupada en México.

\section{El Instituto Mexicano del Seguro Social en el sistema de salud de México}

El sistema de salud mexicano se conforma por el sector público y el privado. El primero incluye a las institucio- nes de seguridad social como el Instituto Mexicano del Seguro Social (IMSS), el Instituto de Seguridad y Servicios Sociales de los Trabajadores del Estado (ISSSTE), Petróleos Mexicanos, Secretaría de Defensa Nacional, Secretaría de Marina, las instituciones de seguridad social para los trabajadores de los gobiernos estatales y las instituciones que atienden a la población sin seguridad social (Secretaría de Salud, Servicios Estatales de Salud, IMSS-Oportunidades y Seguro Popular de Salud). El sector privado abarca a las aseguradoras, hospitales y servicios de atención médica privados. ${ }^{9}$

El IMSS es una institución de seguridad social para los trabajadores y sus familias creada en 1943, con 429000 plazas laborales totales a diciembre de 2010 . En ese mismo periodo contaba con casi 20.3 millones de asegurados en el país, y una población derechohabiente total de más de 52.3 millones, lo que hace que el IMSS sea la institución de seguridad social más grande de América Latina. ${ }^{10}$ Brinda asistencia médica a través de la Dirección de Prestaciones Médicas. La Dirección de Prestaciones Económicas y Sociales otorga servicios sociales necesarios para el bienestar y desarrollo de los asegurados, los beneficiarios y, en algunos casos, la población en general. A través de los servicios de prestaciones económicas, brinda pensiones a los trabajadores jubilados, pensionados o sus beneficiarios y prestaciones en dinero cuando ocurre alguno de los riesgos contemplados en la Ley del Seguro Social, por ejemplo, días subsidiados por incapacidad.

\section{Material y métodos}

\section{Material}

A partir de información censal de la Coordinación de Prestaciones Económicas del IMSS, se obtuvieron datos de sexo, edad, diagnóstico, número total de días por incapacidad, fecha de inicio de las incapacidades, fecha final de las mismas, salario diario integrado y unidad de adscripción de asegurados y beneficiarios del IMSS con diagnóstico de CP (C34), ECV (I60-I69), EPOC (J40-J44)e IAM (I21) de acuerdo con la Clasificación Internacional de Enfermedades (CIE-10).

Ante una incapacidad temporal para el trabajo, la cual se define como la pérdida o disminución por cierto tiempo de las facultades físicas o mentales que imposibilitan al asegurado para realizar su trabajo habitual, ${ }^{11}$ el IMSS expide un certificado, el cual es un documento que brinda justificación ante la ausencia laboral, así como protección financiera al trabajador, ya que a través del mismo se estima la compensación de salario o subsidio que se otorga al trabajador durante el periodo de incapacidad. ${ }^{12}$ 
La base de datos fue depurada a fin de eliminar algunas inconsistencias, como fue el caso de 807 registros de un total de 36 639, en los cuales la variable salario presentaba valores de cero o cercanos a éste por lo que se imputó el salario promedio de los pacientes de la delegación de adscripción a la que el paciente pertenece para el año que correspondía. Todas las variables expresadas en unidades monetarias fueron deflactadas usando el Índice Nacional de Precios al Consumidor y periodo base diciembre de 2009.

El protocolo de este estudio fue revisado por la Comisión de Ética del Instituto Nacional de Salud Pública y fue considerado exento de revisión, debido a que no incluye sujetos humanos, y se trata de análisis de datos que obtuvieron los permisos apropiados en su tiempo y no se proponen cambios que tengan implicaciones éticas.

\section{Métodos}

En coincidencia con los objetivos, el análisis de los costos se hace desde dos enfoques: a) los montos erogados por el IMSS en términos de los subsidios que otorga a las personas por eventos de incapacidad y b) la productividad perdida entre los asegurados del IMSS con un ejercicio de extrapolación a la población ocupada en el país* para el subgrupo mayor de 15 años, mediante la imputación de los parámetros obtenidos en los casos del IMSS.

Con el objeto de estimar los costos atribuibles al consumo de tabaco bajo las perspectivas antes señaladas para las cuatro principales enfermedades asociadas con el consumo de tabaco, se aplicaron las fracciones atribuibles poblacionales ${ }^{13}$ estimadas por Reynales (2007) a los costos calculados, siendo de 0.66 para $\mathrm{CP}, 0.69$ para EPOC, 0.49 para la ECV y de 0.61 para el IAM. ${ }^{14}$ La fracción atribuible poblacional se entiende como la proporción de la enfermedad en la población específica que se eliminaría si no existiera exposición al factor de riesgo, el tabaco en este caso.

\section{a) Montos erogados por el IMSS}

Como se mencionó anteriormente, el IMSS otorga una compensación de salario a los trabajadores en un evento

\footnotetext{
* Total de personas de 12 años y más que realizaron cualquier actividad económica, a cambio de un sueldo, salario, jornal u otro tipo de pago en dinero o especie. Incluye además a las personas que tenían trabajo pero no trabajaron por alguna causa temporal (vacaciones, licencia, enfermedad, mal tiempo, huelga o estaban en espera de iniciar o continuar con las labores agrícolas). Incluye también a las personas que ayudaron en el predio, fábrica, tienda o taller de algún familiar sin recibir sueldo o salario de ninguna especie; y a los aprendices o ayudantes que trabajaron sin remuneración.
}

de incapacidad, por ello se calculan los importes erogados por el instituto de acuerdo con la ecuación 1.

\section{Ecuación 1}

$$
s_{w t}=f a p_{w} * 0.6 \sum_{j=1}^{N}\left(\left(i a c_{j w t}-3\right)\right){ }^{*} i_{j w t}
$$

Donde $s$ es el importe por incapacidad erogado por el IMSS, fap es la fracción atribuible estimada por Reynales (2007), iac son los días de incapacidad acumulados (los días que el trabajador dejó de trabajar debido a la enfermedad), e $i$ es el salario de cotización del trabajador. El subíndice $j$ es el paciente, $w$ el padecimiento y $t$ es el año. Cabe aclarar que el IMSS indemniza las incapacidades a partir del cuarto día y las cubre con $60 \%$ del salario de cotización.

\section{b) Productividad perdida entre los asegurados del IMSS y ejercicio de extrapolación}

La estimación de costos por pérdida de productividad desde el enfoque del capital humano se calculó para los asegurados del IMSS de acuerdo con la ecuación 2:

$$
\begin{gathered}
\text { Ecuación } 2 \\
p p_{w t}=\operatorname{fap}_{w}{ }_{j=1}^{N} \sum_{j=1}^{N}\left(i n c_{j w t}{ }^{*} i_{j w t}\right)
\end{gathered}
$$

Donde $p p$ es la productividad perdida, fap es la fracción atribuible, inc son los días de incapacidad e $i$ es el salario de cotización del trabajador. El subíndice $j$ es el paciente, $w$ es el padecimiento $(C P, E C V, E P O C$ e IAM) y $t$ es el año. El salario fue deflactado usando el Índice Nacional de Precios al Consumidor y tomando como periodo base a diciembre de 2009.

Adiferencia de la cuantificación del inciso anterior, en este caso se asigna un costo desde el primer día de incapacidad y se hace sobre el $100 \%$ del salario de cotización.

A fin de dimensionar este costo en el ámbito nacional, se realizó un ejercicio de extrapolación de los parámetros encontrados en la población asegurada del IMSS a la población ocupada a nivel nacional. Para tal efecto, se obtuvieron datos sobre el número total de asegurados en el IMSS, el ingreso promedio por trabajo de acuerdo con la Encuesta Nacional de Ingresos y Gastos de los Hogares (ENIGH) conducida por el Instituto Nacional de Estadística y Geografía (INEGI) en 2008, ${ }^{15}$ en pesos a precios de 2009 mediante el Índice de Precios al Consumidor, y la población ocupada ${ }^{16}$ mayor a 15 años en México a fin de que corresponda con la edad mínima 
entre los asegurados del IMSS, la cual fue de 16 años. Para expandir el número de días de incapacidad por cada padecimiento (CP, ECV, EPOC e IAM) a la población ocupada mayor de 15 años en el país, se multiplicó el inverso de la proporción de los asegurados del IMSS en la población ocupada en el período 2006-2009 (0.43) por el número de días de incapacidad atribuibles al tabaquismo entre los asegurados del IMSS.

La ecuación 3 ilustra cómo se estimaron los costos en el ámbito nacional causados por los cuatro padecimientos analizados:

Ecuación 3

$$
p p n_{w t}=f a p_{w} * \sum_{j=1}^{N}\left(\operatorname{inc}_{\mathrm{jwt}} *\left(\sum_{1}^{t} I M S S / \sum_{1}^{t} P O\right)^{-1 *} g\right)
$$

Donde $p p n$ es la productividad perdida en el ámbito nacional, fap es la fracción atribuible de la enfermedad, inc son los días de incapacidad correspondiente al IMSS, IMSS es el número de asegurados en el IMSS, PO es la población ocupada y $g$ es el salario diario promedio por trabajo por individuo de acuerdo con la ENIGH 2008, es decir 383.8 pesos a precios de 2009. El subíndice $j$ es el paciente, $w$ es el padecimiento (CP, ECV, EPOC e IAM) $\mathrm{y} t$ es el año (2006-2009).

Este ejercicio de extrapolación de la productividad perdida en trabajadores asegurados en el IMSS a la población ocupada en México implica, principalmente, los siguientes supuestos:

- La prevalencia y días de incapacidad promedio de las enfermedades consideradas son iguales entre asegurados del IMSS y en la población ocupada abierta. Se recurrió a este supuesto ya que se exploraron datos de algunas encuestas (Encuesta Nacional de Salud y Nutrición 2006, entre otras) y no se encontró información suficiente respecto a la prevalencia de dichas enfermedades.

- Los ingresos promedio por trabajo de acuerdo con la ENIGH corresponden a los de la población ocupada y se consideran constantes en términos reales a lo largo del periodo de análisis. Este último supuesto se considera más verosímil que el suponer la igualdad entre el ingreso medio en la población ocupada y los asegurados del IMSS, ya que los salarios del sector formal son más altos que los de la población promedio (incluye informales).

\section{Resultados}

En el cuadro I se presenta un análisis descriptivo de los pacientes del IMSS diagnosticados con las enfer- medades analizadas. Puede apreciarse que la mayor parte de estos son hombres, ya que en ninguno de los cuatro años analizados el porcentaje fue menor de 74 . La edad promedio de los pacientes fue un poco menor de 50 años para el total de registros, que fueron aproximadamente 41000 en los cuatro años de análisis, con una desviación estándar de poco más de once años. Por otro lado, el número de pacientes es menor al número de registros puesto que podían existir individuos con más de un registro de incapacidad. Además, se muestra la distribución de los pacientes por región-DF y resto del país-. Resalta que la participación del DF para los cuatro padecimientos considerados en este estudio ha disminuido en el último año del análisis; en 2006 era de $14.3 \%$, para 2007 fue de $15.3 \%$, en 2008 fue de $14.1 \%$ y finalmente en 2009 disminuyó a 11.5\%.

\section{a) Montos erogados por el IMSS atribuibles al tabaquismo}

El cuadro II muestra los montos erogados por el IMSS como subsidio por incapacidad de acuerdo con la ecuación 1. Los montos totales atribuibles al tabaquismo en el periodo ascendieron a 143.9 millones de pesos. El padecimiento más costoso es el IAM, con 106.1 millones de pesos, y el menos costoso es el cáncer pulmonar, ya que ese instituto erogó 2.03 millones de pesos.

Sin embargo, el panorama es diferente si analizamos el costo promedio por paciente. El cáncer pulmonar resulta ser el más elevado, con 18178 pesos promedio

Cuadro I

ANÁLISIS DESCRIPTIVO DE LOS PACIENTES CON DISCAPACIDAD POR CÁNCER PULMONAR, ENFERMEDAD CEREBROVASCULAR, ENFERMEDAD PULMONAR OBSTRUCTIVA CRÓNICA E INFARTO AGUDO DE MIOCARDIO. MÉXICO, IMSS, 2006-2009'

\begin{tabular}{lrrrr} 
& 2006 & 2007 & 2008 & 2009 \\
\cline { 2 - 5 } & & & & \\
Registros totales & 8986 & 10538 & 10965 & 10409 \\
\hline Pacientes* Distrito Federal & $\mathrm{I} 128$ & $\mathrm{I} 417$ & $\mathrm{I} 387$ & $\mathrm{I} 103$ \\
\hline Pacientes resto del país & 6760 & 7854 & 8468 & 8522 \\
\hline Pacientes totales & 7888 & $927 \mathrm{I}$ & 9855 & 9625 \\
\hline Hombres (\%) & 76.1 & 75.8 & 75.4 & 74.4 \\
\hline Mujeres (\%) & 23.9 & 24.2 & 24.6 & 25.6 \\
\hline Edad media (años) & 49.6 & 49.6 & 49.7 & 49.5 \\
\hline Desviación estándar & 11.5 & 11.3 & 11.3 & 11.5 \\
\hline
\end{tabular}

* Considerando que los números de seguridad social son únicos Fuente: Elaboración propia con base en datos de la Coordinación de Prestaciones Sociales y Coordinación de Prestaciones Económicas del IMSS, 2006-2009 
Cuadro II

Montos ATRIBUibles erogados POR EL IMSS PARA los CASOS QUE CUMPLIERON CON LOS SUPUESTOS PARA SER BENEFICIARIOS POR REGIÓN (\$ 2009). MÉXICO, 2006-2009

\begin{tabular}{|c|c|c|c|c|c|}
\hline \multirow{2}{*}{\multicolumn{2}{|c|}{ Zona/Enfermedad }} & \multicolumn{4}{|c|}{ Año } \\
\hline & & 2006 & 2007 & 2008 & 2009 \\
\hline \multicolumn{6}{|c|}{ Distrito Federal } \\
\hline \multirow[t]{5}{*}{$\mathrm{CP}$} & \# Pacientes & 3 & 5 & 2 & 3 \\
\hline & Cambio (\%) & & 40 & -150 & 33.3 \\
\hline & Días de incapacidad totales & 293 & 269 & 283 & 360 \\
\hline & Cambio (\%) & & -8.9 & 4.9 & 21.4 \\
\hline & Montos erogados totales $(\$ 2009)$ & 93074 & 68437 & 28095 & 194878 \\
\hline \multirow[t]{5}{*}{ ECV } & \# Pacientes & 49 & 58 & 59 & 49 \\
\hline & Cambio (\%) & & 15.5 & 1.7 & -20.4 \\
\hline & Días de incapacidad totales & 5266 & 7419 & 6319 & 3628 \\
\hline & Cambio (\%) & & 29 & -17.4 & -74.2 \\
\hline & Montos erogados totales $(\$ 2009)$ & $103 \mid 265$ & $|188| 3 \mid$ & 837109 & 608530 \\
\hline \multirow[t]{5}{*}{ EPOC } & \# Pacientes & 115 & 170 & 167 & 156 \\
\hline & Cambio (\%) & & 32.4 & -1.8 & -7.1 \\
\hline & Días de incapacidad totales & 2803 & 5069 & 5517 & 3054 \\
\hline & Cambio (\%) & & 44.7 & 8.1 & -80.6 \\
\hline & Montos erogados totales $(\$ 2009)$ & 342317 & 946724 & I 057217 & 530012 \\
\hline \multirow[t]{5}{*}{ IAM } & \# Pacientes & 331 & 356 & 340 & 267 \\
\hline & Cambio (\%) & & 7 & -4.7 & -27.3 \\
\hline & Días de incapacidad totales & 25525 & 22321 & 22916 & 13387 \\
\hline & Cambio (\%) & & -14.4 & 2.6 & -71.2 \\
\hline & Montos erogados totales $(\$ 2009)$ & 5003625 & 4435290 & 4168914 & 2473768 \\
\hline \multirow[t]{5}{*}{ Sub-total } & \# Pacientes & 499 & 588 & 568 & 476 \\
\hline & Cambio (\%) & & I5.I & -3.5 & -19.3 \\
\hline & Días de incapacidad totales & 33887 & 35078 & 35035 & 20429 \\
\hline & Cambio (\%) & & 3.4 & -0.1 & -71.5 \\
\hline & Montos erogados totales $(\$ 2009)$ & 6470281 & 6638583 & 6091336 & 3807187 \\
\hline
\end{tabular}

Resto del país

\begin{tabular}{|c|c|c|c|c|c|}
\hline $\mathrm{CP}$ & \# Pacientes & 24 & 22 & 22 & 30 \\
\hline & Cambio (\%) & & -9.1 & 0 & 26.7 \\
\hline & Días de incapacidad totales & 2957 & 2328 & 2873 & 2286 \\
\hline & Cambio (\%) & & -27 & 19 & -25.7 \\
\hline & Montos erogados totales $(\$ 2009)$ & 485497 & 331110 & 514920 & 3|| $55 \mid$ \\
\hline ECV & \# Pacientes & 349 & 431 & 464 & 457 \\
\hline & Cambio (\%) & & 19 & 7.1 & -1.5 \\
\hline & Días de incapacidad totales & 35275 & 40355 & 45423 & 27825 \\
\hline & Cambio (\%) & & 12.6 & 11.2 & -63.2 \\
\hline & Montos erogados totales $(\$ 2009)$ & 4988262 & 6084653 & 6911840 & 3649102 \\
\hline
\end{tabular}


Continuación..

\begin{tabular}{|c|c|c|c|c|c|}
\hline EPOC & \# Pacientes & 593 & 730 & 760 & 945 \\
\hline & Cambio (\%) & & 18.8 & 3.9 & 19.6 \\
\hline & Días de incapacidad totales & 16893 & 19274 & 22297 & 18137 \\
\hline & Cambio (\%) & & 12.4 & 13.6 & -22.9 \\
\hline & Montos erogados totales ( $\$ 2009)$ & 496728 & 1896466 & 2291361 & | 867365 \\
\hline
\end{tabular}

\begin{tabular}{|c|c|c|c|c|c|}
\hline IAM & \# Pacientes & 1835 & 2062 & 2209 & 2049 \\
\hline & Cambio (\%) & & II & 6.7 & -7.8 \\
\hline & Días de incapacidad totales & 147676 & 161275 & 174625 & 109398 \\
\hline & Cambio (\%) & & 8.4 & 7.6 & -59.6 \\
\hline & Montos erogados totales $(\$ 2009)$ & 22685258 & 24232341 & 26053159 & 17088248 \\
\hline
\end{tabular}

\begin{tabular}{|c|c|c|c|c|c|}
\hline Sub-total & \# Pacientes & 2802 & 3245 & 3455 & 3481 \\
\hline & Cambio (\%) & & 13.7 & 6.1 & 0.7 \\
\hline & Días de incapacidad totales & 202802 & 223233 & 245219 & 157646 \\
\hline & Cambio (\%) & & 9.2 & 9 & -55.6 \\
\hline & Montos erogados totales $(\$ 2009)$ & 29655746 & 32544570 & $3577 \mid 280$ & 22916267 \\
\hline
\end{tabular}

\begin{tabular}{|c|c|c|c|c|c|}
\hline Total & \# Pacientes & 3301 & 3833 & 4023 & 3957 \\
\hline & Cambio (\%) & & 13.9 & 4.7 & -1.7 \\
\hline & Días de incapacidad totales & 236689 & 258311 & 280254 & 178075 \\
\hline & Cambio (\%) & & 8.4 & 7.8 & -57.4 \\
\hline & Montos erogados totales $(\$ 2009)$ & 36126027 & 39183152 & 41862617 & 26723454 \\
\hline
\end{tabular}

CP: cáncer pulmonar; ECV: enfermedad cerebrovascular; EPOC: enfermedad pulmonar obstructiva crónica; IAM: infarto agudo de miocardio Fuente: Elaboración propia con base en datos de la Coordinación de Prestaciones Sociales y Coordinación de Prestaciones Económicas del IMSS, 2006-2009

por paciente, mientras que la EPOC es el padecimiento menos costoso en estos términos, con 2869 pesos en promedio por paciente. Por otro lado, en el período de análisis, los montos erogados para subsidiar las incapacidades por cáncer pulmonar en el Distrito Federal respecto al total del país representan 19\%, cifra que es más proporcional que su participación lo que podría ser causado por el mayor costo de oportunidad de sus trabajadores. Asimismo, ese porcentaje fue de 14.5, 27.6 y $15.2 \%$ para ECV, EPOC e IAM, respectivamente. Es importante hacer notar que el porcentaje que representan las indemnizaciones en el Distrito Federal ha disminuido, en correspondencia con la reducción en su participación en el número de casos por año, como se comentó anteriormente, y en 2009 fue de 14.3, 22.1 y $12.6 \%$ para ECV, EPOC e IAM, respectivamente.

\section{b) Productividad perdida entre los asegurados del IMSS y en la población ocupada en México}

El cuadro III muestra la productividad perdida atribuida al tabaquismo a causa de CP, ECV, EPOC e IAM entre los asegurados del IMSS debido a los días de incapacidad de los asegurados, entre 2006 y 2009, el cual fue de $298.2 \mathrm{mi}-$ llones en total. Asimismo, esto representa casi un millón 140000 días-hombre perdidos atribuibles al consumo de tabaco debido a las incapacidades ocasionadas por las cuatro enfermedades consideradas en el estudio.

Por otro lado, la figura 1 muestra la estimación de los costos por pérdida de productividad atribuible por días de incapacidad en la población ocupada mayor de 15 años en México debido a las cuatro enfermedades analizadas en este estudio de acuerdo con la ecuación 3. En el periodo de análisis, la estimación de la productividad perdida total a causa de estas enfermedades es de 733.9 millones de pesos. De estos, 437.8 millones pueden atribuirse al tabaquismo. Además, se estima una pérdida atribuible al tabaquismo de 2.65 millones de días-hombre.

\section{Discusión}

Este estudio encontró que el tabaquismo ocasiona pérdidas cuantiosas en términos de subsidios y productividad. De 2006 a 2009 se erogaron 143.9 millones de pesos 


\section{Cuadro III}

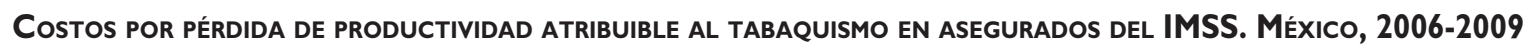

\begin{tabular}{|c|c|c|c|c|c|}
\hline \multirow{2}{*}{\multicolumn{2}{|c|}{ Zona/ Enfermedad }} & \multicolumn{4}{|c|}{ Año } \\
\hline & & 2006 & 2007 & 2008 & 2009 \\
\hline \multicolumn{6}{|c|}{ Distrito Federal } \\
\hline \multirow[t]{3}{*}{$\mathrm{CP}$} & Pacientes & 3 & 5 & 3 & 4 \\
\hline & Días de incapacidad & 293 & 269 & 284 & 592 \\
\hline & Costo totales $(\$ 2009)$ & 156746 & 121830 & 56074 & 203892 \\
\hline \multirow[t]{3}{*}{ ECV } & Pacientes & 57 & 71 & 75 & 61 \\
\hline & Días de incapacidad & 5791 & 7479 & 6883 & 6338 \\
\hline & Costo totales $(\$ 2009)$ & 1867312 & 2301305 & I 526987 & I 430807 \\
\hline \multirow[t]{3}{*}{ EPOC } & Pacientes & 234 & 345 & 342 & 293 \\
\hline & Días de incapacidad & 3122 & 5576 & 5957 & 4617 \\
\hline & Costo totales $(\$ 2009)$ & 730863 & I 833263 & 1965679 & 993985 \\
\hline \multirow[t]{3}{*}{ IAM } & Pacientes & 409 & 470 & 451 & 348 \\
\hline & Días de incapacidad & 26170 & 23417 & $24|5|$ & 21477 \\
\hline & Costo totales $(\$ 2009)$ & 9149863 & 8312606 & 7740035 & 5955699 \\
\hline \multirow[t]{3}{*}{ Sub-total } & Pacientes & 704 & 890 & 871 & 705 \\
\hline & Días de incapacidad & 35376 & 36741 & 37274 & 33023 \\
\hline & Costo totales $(\$ 2009)$ & II 904785 & 12569003 & II 288776 & 8584384 \\
\hline
\end{tabular}

Resto del país

\begin{tabular}{llrrrr} 
CP & Pacientes & 28 & 26 & 26 & 36 \\
\hline Días de incapacidad & 3050 & 2364 & 3014 & 4033 \\
\hline Costo totales $(\$ 2009)$ & 905053 & 577682 & 888840 & 1387772 &
\end{tabular}

\begin{tabular}{llrrrr} 
ECV & Pacientes & 447 & 541 & 596 & 598 \\
\hline Días de incapacidad & 38260 & 44047 & 50252 & 53478 \\
\hline Costo totales $(\$ 2009)$ & 9317410 & 11120321 & 13121172 & 12072466
\end{tabular}

\begin{tabular}{llrrrr} 
EPOC & Pacientes & I 258 & I 493 & I 615 & I 846 \\
\hline Días de incapacidad & 19833 & 22227 & 25538 & 29040 \\
\hline Costo totales $(\$ 2009)$ & 3467477 & 4168311 & 4760370 & 6252707
\end{tabular}

\begin{tabular}{llrrrr} 
IAM & Pacientes & 2446 & 2797 & 2987 & 2811 \\
\hline Días de incapacidad & 159021 & 174942 & 189149 & 180213 \\
\hline Costo totales $(\$ 2009)$ & 42083208 & 45266491 & 48515755 & 49975235
\end{tabular}

\begin{tabular}{llrrrr} 
Sub-total & Pacientes & 4179 & 4857 & 5225 & 5291 \\
\hline & Días de incapacidad & 220163 & 243580 & 267953 & 266764 \\
\hline & Costo totales $(\$ 2009)$ & 55773148 & 61132805 & 67286137 & 69688180 \\
Total & Pacientes & 4883 & 5747 & 6095 & 5996 \\
\hline & Días de incapacidad & 255539 & 280321 & 305227 & 299788 \\
\hline & Costo totales $(\$ 2009)$ & 67677933 & 73701808 & 78574912 & 78272564
\end{tabular}

CP: cáncer pulmonar; ECV: enfermedad cerebrovascular; EPOC: enfermedad pulmonar obstructiva crónica; IAM: infarto agudo de miocardio Fuente: Elaboración propia con base en datos de la Coordinación de Prestaciones Sociales y Coordinación de Prestaciones Económicas del IMSS, 2006-2009 


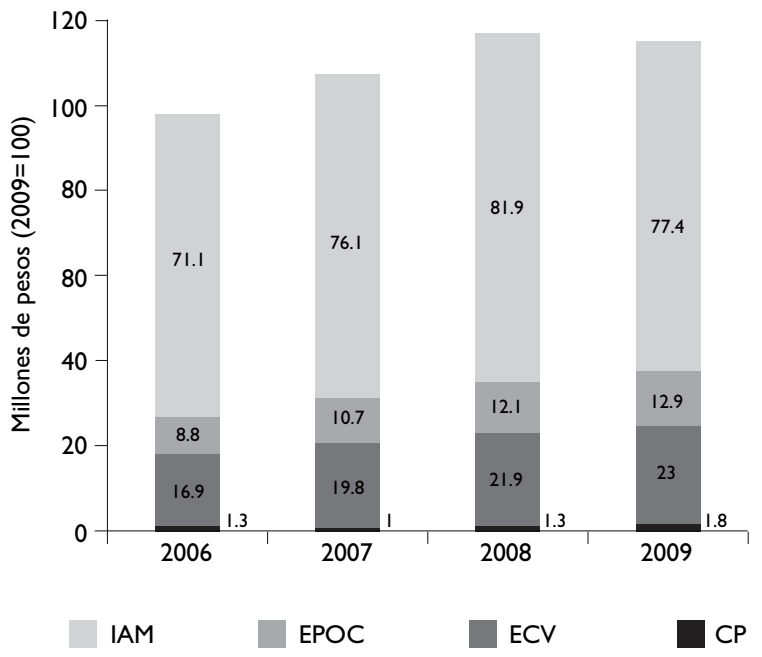

Fuente: elaboración propia con base en datos de la Coordinación de Prestaciones Sociales y Coordinación de Prestaciones Económicas del IMSS, Encuesta Nacional de Ocupación y Empleo 2006-2009

Figura I. Costos por pérdida de PROductividad POR ENFERMEDADES ATRIBUIBLES AL TABAQUISMO EN LA POBLACIÓN OCUPADA MAYOR DE I5 AÑos. MÉXICO, 2006-2009

de 2009 para subsidiar las incapacidades en el IMSS. Adicionalmente, estas incapacidades ocasionaron 298.2 millones de pesos en pérdidas por ausencia laboral, lo que implicaría 437.8 millones de pesos si se trasladara a toda la población ocupada en México, según nuestra estimación. Esta información constituye un elemento adicional para reforzar las políticas públicas que buscan controlar el consumo de tabaco, de acuerdo con el Convenio Marco para el Control del Tabaco, ratificado por México en 2004.

Este documento cuantifica los costos ocasionados únicamente por la pérdida de productividad utilizando datos censales del IMSS lo que representa su principal fortaleza. Sin embargo, no estima los costos de atención médica ni los intangibles como el dolor y sufrimiento ocasionados, y las afectaciones a familiares. Excluye también los costos causados por años de vida perdidos en México. ${ }^{*}$ De manera similar, se toman en cuenta únicamente los días de ausentismo laboral, pero no la disminución en productividad en trabajadores presentes en su empleo pero que no se desempeñan conforme a su capacidad normal debido a una enfermedad. ${ }^{17}$

\footnotetext{
* Comisión Federal de Mejora Regulatoria. El tabaquismo en México: Análisis y Recomendaciones de Mejora Regulatoria. (No publicado) México, 2011.
}

Además, es necesario cobrar conciencia de los resultados diferentes cuando se usa el enfoque del capital humano en comparación con el método de costos friccionales. Este último consiste en considerar a la productividad perdida como el tiempo que transcurre entre la ausencia de un trabajador y la llegada de un remplazo. Usualmente, las estimaciones de costos friccionales son de entre 1 y $3 \%$ de las estimaciones realizadas mediante el método del capital humano. ${ }^{18}$

Por otra parte, existe una aparente disminución relativa en las incapacidades ocasionadas por las enfermedades relacionadas con el consumo de tabaco en el Distrito Federal respecto al resto del país, sobre todo en 2009, lo que contrasta con la estabilidad del porcentaje de asegurados de las delegaciones del Distrito Federal respecto al país ${ }^{19}(18.2,18.4,18.1$ y $18.5 \%$ en 2006,2007 , 2008 y 2009, respectivamente). Es necesario llevar a cabo una investigación más profunda al respecto para explicar esta disminución y la posible influencia de legislaciones que promueven ambientes libres de humo de tabaco, tales como la Ley de Protección a la Salud de los No Fumadores del Distrito Federal que entró en vigor en abril de 2008.

Es preciso poner en perspectiva estos hallazgos, de tal forma que se aprecie la dimensión del problema expuesto aquí. Por ejemplo, se encontró que para 2006, los días de incapacidad atribuibles al tabaco por las cuatro enfermedades consideradas en este estudio representan $0.96 \%$ de los días subsidiados por enfermedad general en el IMSS. Este indicador es de 1.02, 1.08 y $0.66 \%$ para 2007, 2008 y 2009, respectivamente.

Por otro lado, los montos erogados como subsidio por el IMSS atribuibles al tabaquismo para las cuatro enfermedades respecto a los gastos por subsidios por enfermedad general del instituto fueron de 1.12, 1.15, 1.19 y $0.74 \%$ para el periodo 2006 a 2009, respectivamente. ${ }^{20}$

Finalmente, los montos erogados por el IMSS atribuibles al tabaquismo, por concepto de incapacidad para las cuatro enfermedades, respecto a los gastos de operación del instituto son de $0.02,0.02,0.2$ y $0.1 \%$ para 2006, 2007, 2008 y 2009, respectivamente. ${ }^{10}$ Reynales (2007) reportó que los costos de atención médica atribuibles al consumo de tabaco para CP, ECV, EPOC e IAM fueron de 4.3\% de los gastos de operación del IMSS en 2004.

\section{Conclusiones}

Los hallazgos de este estudio se unen a la amplia evidencia que demuestra los importantes costos económicos y sociales que la epidemia del tabaquismo ocasiona. Este escenario hace prioritaria la implementación y fortalecimiento de políticas públicas en el sector encaminadas a reducir las pérdidas de bienestar social. 
En este sentido, existen políticas impulsadas por la Secretaría de Salud y expresadas en el Programa Nacional de Salud 2007-2012 en lo relativo al combate de las adicciones, tales como el tabaco, alcohol y sustancias psicoactivas. ${ }^{21}$ Estas medidas buscan alinearse con el Convenio Marco para el Control del Tabaco, de la Organización Mundial de la Salud y atacan al problema del consumo de tabaco desde el lado de la oferta y la demanda. Particularmente y sin dejar del lado el resto de artículos y medidas promovidas por el mismo, nos referimos al Artículo 6 que hace referencia a las medidas relacionadas con los precios e impuestos para reducir la demanda del tabaco; al Artículo 8, que obliga a los países signatarios a adoptar y aplicar medidas legislativas efectivas de protección contra la exposición al humo de tabaco en lugares de trabajo interiores, medios de transporte, lugares públicos cerrados; al Artículo 11 que versa sobre el empaquetado y etiquetado de los productos de tabaco; al Artículo 13, sobre la prohibición de la publicidad, promoción y patrocinio del tabaco; al Artículo 14, acerca de medidas de reducción de la demanda relativas a la dependencia y al abandono del tabaco; al Artículo 15, sobre el comercio ilícito de productos de tabaco y finalmente al Artículo 20, que promueve la investigación, vigilancia e intercambio de información. ${ }^{22}$

\section{Agradecimientos}

Agradecemos a la Coordinación de Prestaciones Económicas del IMSS por facilitar la base de datos necesaria para llevar a cabo este estudio. Estos resultados forman parte del proyecto Investigación de Economía del Tabaco para apoyar el Control del Tabaco en México, apoyado financieramente por la Iniciativa Global Bloomberg para Reducir el Consumo de Tabaco.

Declaración de conflicto de intereses: Los autores declararon no tener conflicto de intereses.

\section{Referencias}

I. Warner K, Hodgson TA, Carroll C. Medical cost of smoking in the United States: Estimates, their validity, and their implications. Tob Control 1999; (8): 290-300.

2. Leistikow B. The Human and Financial Costs of smoking. En: Smoking and Pulmonary and Cardiovascular Diseases. Clin Chest Med 2000; (21):189-195.

3. Samet JM. The risk of active and passive smoking. En: Slovic P ed. Smoking: Risk, perception and policy. Thousand Oaks, California: Sage Publications, 2002.
4. Easton B. The social costs of tobacco use and alcohol misuse. Wellington: Public Health Monograph Series, 1997.

5. Cordeiro de Azevedo AB, Bosi-Ferraz, M, Mesquita-Ciconelli R. Indirect Costs of Rheumatoid Arthritis in Brazil. Value in Health 2008; II(5): 869-877.

6. Hamer HM, Spottke A, Aletsee C, Knake S, Reis J, Strzelczyk A et al. Direct and indirect costs of refractory epilepsy in a tertiary epilepsy center in Germany. Epilepsia 2006; 47(I2):2I65-2I72

7. Xie F, Thumboo J, Fong KY, Lo NN, Yeo SJ, Yang KY et al. A study on indirect and intangible costs for patients with knee osteoarthritis in Singapore. Value in Health 2008; II(SI):S84-S90.

8. Tsai SP, Wen CP, Hu SC, Cheng TY, Huang SJ. Workplace smoking related absenteeism and productivity costs in Taiwan. Tob Control 2005; (14):i33-i37.

9. Frenk J, Gómez-Dantés O. Para entender el sistema de salud de México. México, DF: Nostra Ediciones, 2008:60.

10. Dirección de Finanzas. Instituto Mexicano del Seguro Social. Reporte de gestión nacional 52. Información de enero de 2006 a diciembre de 2010. México: IMSS, 2010.

II. Instituto Mexicano del Seguro Social. Reglamento de Servicios Médicos. Capítulo VI: De la expedición de certificados de incapacidad temporal para el trabajo a los asegurados del régimen obligatorio. [Consultado 2011 noviembre 16]. Disponible en http://www.dvvimss.org. $\mathrm{mx} / \mathrm{pdf} /$ reglamentodeserviciosmedicosdelimss.pdf.

12. Constantino-Casas P, Torres-Arreola LP, Posadas-García JL, Neváres-Sida A, García-Contreras F. Prescripción de certificados de incapacidad temporal en el IMSS. Rev Med Inst Mex Seguro Soc 2007; 45 (I): 89-96.

13. Yang MC, Fann CY, Wen CP, Wen CP, Cheng TY. Smoking attributable medical expenditures, years of potential life lost, and the cost of premature death in Taiwan. Tob Control 2005; 14: i62-i70.

14. Reynales-Shigematsu LM. Costos de atención por enfermedades atribuidas al consumo de tabaco en el Instituto Mexicano del Seguro Social. Salud Publica Mex 2007; 49:EI 9-22.

15. Instituto Nacional de Estadística y Geografía. Encuesta Nacional de Ingresos y Gastos de los Hogares. 2008. [Consultado 2010 junio 13]. Disponible en: http://www.inegi.org.mx/est/contenidos/espanol/soc/sis/ microdatos/enigh/default.aspx?s=est\&c $=\mid 4606$

16. Instituto Nacional de Estadística y Geografía. Encuesta Nacional de Empleo. [Consultado 2010 abril 8]. Disponible en: http://www.inegi. org.mx/est/contenidos/espanol/proyectos/encuestas/hogares/enoe/bd/ consulta2/po.asp?s=est $\& c=10826$

17. Trogdon JG, Finkelstein EA, Hylands T, Dellea PS, Kamal-Bahl SJ. Indirect costs of obesity: a review of the current literature. Journal Compilation. International Association for the study of obesity. Obes Rev 2008; (9): 489-500.

18. Moller L, Matic S (eds). Best practice in estimating the costs of alcohol. Recommendations for future Studies. Ginebra: WHO Europe, 2010.

19. Instituto Mexicano del Seguro Social. Memoria Estadística 2010. [Consultado 20II diciembre 18]. Disponible en: http://www.imss.gob.mx/ estadisticas/financieras/Pages/memoriaestadistica.aspx

20. Sistema de información Delegacional. Subdirección de Prestaciones Económicas. México: IMSS, 2010.

21. Secretaría de Salud. Programa Nacional de Salud 2007-20I2. Por un México sano: construyendo alianzas para una mejor salud. México: Secretaría de Salud, 2007.

22. Organización Mundial de la Salud. Convenio Marco para el Control del Tabaco. Ginebra: OMS, 2005. 